\title{
Performance Analysis of Ubiquitous Wireless Connectivity in a 3G/IEEE 802.11 Integrated Network
}

\author{
Abdessadeq Fettouh \\ LISI Laboratory \\ Cadi Ayyad University \\ Marrakech, MOROCCO
}

\author{
Najib El Kamoun \\ STIC Laboratory \\ Chouaib Doukkali University, \\ B.P: 20 El Jadida MOROCCO
}

\author{
Abdelaziz El Fazziki \\ LISI Laboratory \\ Cadi Ayyad University \\ Marrakech, MOROCCO
}

\begin{abstract}
UMTS networks promise to offer always on, ubiquitous connectivity with relatively low data rates for moderate to high mobility users in macro cell environments. On the other hand, IEEE 802.11 (WLAN) offers much higher data rates, comparable to $3 \mathrm{G}$ and other wireless local area networks, to users with low mobility characteristics in smaller cell environments. Where UMTS/WLAN networks overlap, a dual-mode UMTS/WLAN terminal could access high bandwidth data services where WLAN coverage is offered and use UMTS services elsewhere. This leads to fundamental questions on how UMTS and WLAN can be integrated, It were build two different scenarios, one for loose coupling scheme and the other one for open coupling scheme. In loose coupling scheme both billing and authentication is common for UMTS and WLAN networks while in case of open coupling scheme only billing is common for WLAN and UMTS where as they use there own separate authentication servers. The OPNET simulated results reveal that the loose coupling architecture performance is better than the open coupling architecture for all the applied applications and measurement parameters during the Mobility between WLAN and UMTS.
\end{abstract}

\section{General Terms}

Mobility Management.

\section{Keywords}

Vertical Handover, IEEE 802.11, 3G, Ubiquitous Connectivity.

\section{INTRODUCTION}

More public services available online and an expectation that services will be accessible at all times via a variety of different devices driven increasingly by a sense of the users choice. In this article it is considered two particular complementary technologies: UMTS (Universal Mobile Telecommunications System) and WLAN (wireless local area networks). In order to navigate between these two technologies in a seamless fashion, a specific interconnection architecture has been chosen, namely the tight coupling architecture. The current work concentrates on the mobility management for support of the QoS (Quality of Service) of the non real time Applications in heterogeneous network UMTS/WLAN.

This study should serve as a basis to facilitate the adequate choice of the most efficient architecture. Interoperability between WLAN network and the UMTS network is very critical, because it can not be ot afford to have a degradation of performance which is due to the integration between the two systems mentioned above. Previous research has shown that there are enough problems to solve in order to ensure ubiquitous connectivity in heterogeneous networks, it was chosen this modeling for this reason, in order to get rid of difficulties that may be arise from the architecture systems for UMTS / WLAN

\section{RELATED WORKS}

Many Researches was performed by different researchers regarding the integration of $3 \mathrm{G}$ and WLAN networks.

Tsao et al. [12], Integration Approaches like gateway, Mobile IP and emulator schemes were analyzed and concluded that Mobile IP is the simplest way to achieve the 3G/WLAN network integration. Another reason for choosing Mobile IP, is that its deployment in various networks independently and because mobile IP is becoming an available standards that. However, the Mobile IP approach is not an adequate solution for the real time Applications as handover delay is bigger than in the gateway approach is. However, the mobility of services and applications is supported. Generally the emulator approach was defined as most difficult approach among the three approaches mentioned above as networks are tightly coupled; however, it provides the lowest handover delay among all of the examined approaches.

n [13], Apostolic Approach of some 3G-WLAN integration schemes which will provide high performance for $3 \mathrm{G}$ subscribers.

In [19], Fauzi and Mohammad proposed method for WLANUMTS integration and debated a protocol to save resources for Handover.

In [14], F. Siddiqui et al. suggested and applied architecture for a bi-mode mobile terminal that moves its active sessions, and analyzed handover performance in between UMTS and WLAN networks.

In [15], Yu Zhou et al. proposed a dual-mode mobile terminal module for the integrated UMTS-WLAN network and suggested an algorithm based on network access selection.

[16], for a tightly coupled GRPS-WLAN network the authors suggested an authentication scheme of a mobile node in a heterogeneous wireless during the vertical handover.

M. Shi et.al [17], develop an agent based scheme for a WLAN-cellular network. This scheme provides significant authentication for billing, all this without a peer to-peer roaming agreements between different wireless networks.

In contrast to the above mentioned research efforts, the prime concern of this paper is to study the effects of two open coupling and Loose coupling architecture variations on different applications and services. 


\section{Proposed Architectures}

All current architectures in proposed wireless heterogeneous networks were deployed in an assumption that the network layer protocol used is IP. Applications and Transport layers are also IP layer compatible. The intention of WLAN-UMTS interworking is to extend the services capabilities of UMTS network also to WLAN environment and vice versa.

\section{WLAN/3G Architectures Integration}

Several ways have been proposed in the literature in order to classify the different interworking architectures [2-8]. Although the same terms are usually used in these classifications, there are important deviations in their meaning. The first standardization effort towards the categorization of solutions has been made by ETSI [2]. There, "loose coupling" indicates a way of interconnecting independently the two networks, utilizing only a common subscription. Moreover, "tight coupling" suggests that WLAN appears as another access network to the cellular core network, thus, both data and signaling traffic is transferred through the cellular network.

\section{Tight Coupling}

Is an architecture where WLAN network is directly attached UMTS network (either GGSN or SGSN) influent the functionality of some UMTS component. Such method can apply part of the UMTS feature at the integration point or apply Mobile IP solution, offering soft or hard handovers respectively. The mobile-controlled and network-controlled handovers are exclusively used as these comply with the respective handover types existing in UMTS [1].

\section{Very Tight Coupling}

Very tight coupling schemes focus on interworking at the UTRAN level and, more precisely, on incorporating RNC or lower UMTS entities' functionality into WLAN components. These solutions can perform soft handover and take the handover decision as in the tight coupling case. Very tight coupling is considered quite complicated and only few solutions have been proposed in this area.

\section{Loose Coupling}

In [9] in the loose coupling architecture, networks are implemented and joined to each other independently. From the UMTS network point of view, the central point of the interconnection is located after the Gateway GPRS Support Node (GGSN), as shown in the Fig. 1. Therefore, WLAN network avoid the UMTS core network for establishing a direct connection with the external PDNs and simultaneously keep an indirect connection to the UMTS network. Mobile IP is used to guaranty the mobility in the networks (Lampropoulos, 2007).

To provide the interconnection between UMTS and WLAN networks, WLAN gateway is a device that is required for roaming purposes. This gateway is support billing and authentication. The gateway is connected to UMTS AAA server which in turn connects it with the internet. Hence, no direct connection between UMTS and WLAN exists. After that, WLAN data traffic goes directly through the internet instead of passing through the UMTS core network. This method helps a UMTS service provider to track accounting records of WLAN and ease the billing operation for both integrated networks. If the loose coupling scheme is used, it allows the autonomous deployment and operation of networks. This enables the network service providers to profit from the existence of other providers' Networks. Moreover, minimal improvement is required in existing network infrastructure; hence the deployment of the integration is not so expensive. Subscribers can get access to wireless access networks while subscribing to only one service provider.

\section{Authentication, Authorization, and Accounting}

AAA management is very important in WLAN-UMTS integrated network. Authentication is the act of making lawful, in the form of a pre-existing label from a mutually known namespace, as the originator of the message (message authentication) or as the channel end point [10]. Authorization is an official permission or approval. This particular permission can be, for example, an access to a resource [10]. Accounting is the act of collecting resource utilization data for predicting future events, capacity planning, billing, auditing, and cost allocation. These ways to behave are applied to all kinds of networks, especially to $3 \mathrm{G}$ Wireless infrastructures targeted to public networks such as UMTS as well as wireless local area networks like [11], but UMTS and WLAN networks have different databases management systems and AAA process.

\section{Billing}

Services offered by different network operators can be billed in combined manner, is one of the motivations that justifying the interworking of UMTS and WLAN, which means that a subscriber will have a single combine bill. This implies that the internal network of the subscriber will be responsible for collecting the billing information and summarizing them together. Two billing forms are available; pre-paid and postpaid options. In prepaid option, a subscriber paid a certain amount of money in advance. When the subscriber utilizes network services, the home network operator verifies the charging information and subtract corresponding amount of money from the subscriber's credit. When the subscriber is out of credit access network services is denied. In post-paid billing, a subscriber has a billing contract with the home service provider to pay for a period of time generally each month at intervals and is charged regularly for the usage within this period.

\section{Simulation Scenarios and Discussions}

The utilization of WLAN is defined as a packet based access network complementary to UMTS networks. It has been integrated into the UMTS network so that both Billing and Authentication is common for UMTS and WLAN; this is the open coupling architecture. In the other case of loose coupling, which allows WLAN to bypass the $3 \mathrm{G}$ core network and interface directly to the core IP network, only the billing Server is common for both networks WLAN and UMTS, each one use its own separate authentication servers. Network design for the two scenarios is shown in the figures 2 and 3 below. Those approaches completely separate the data paths in the WLAN and UMTS networks. Therefore the WLAN data traffic is never injected into the UMTS core network. It allows for independent deployment of WLAN and UMTS networks and roaming agreement can be established between the operators of the two networks. Therefore a minimal modification to UMTS network was required. Mobile IP was used for mobility management of mobile users. 


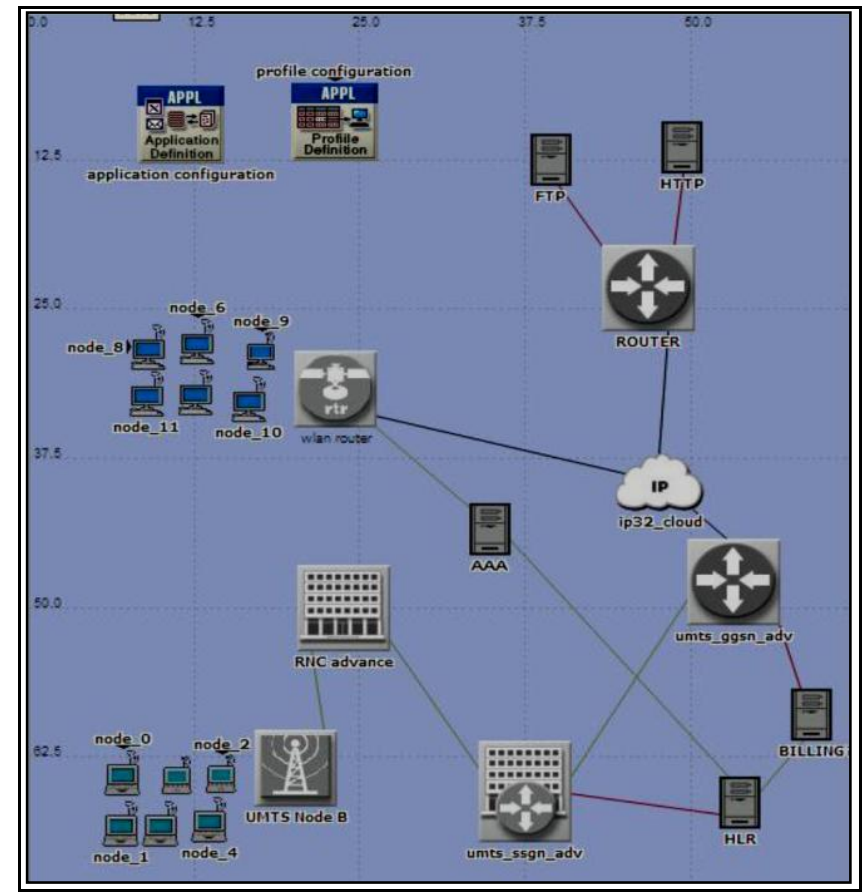

Fig 2: Loose coupling scheme architecture

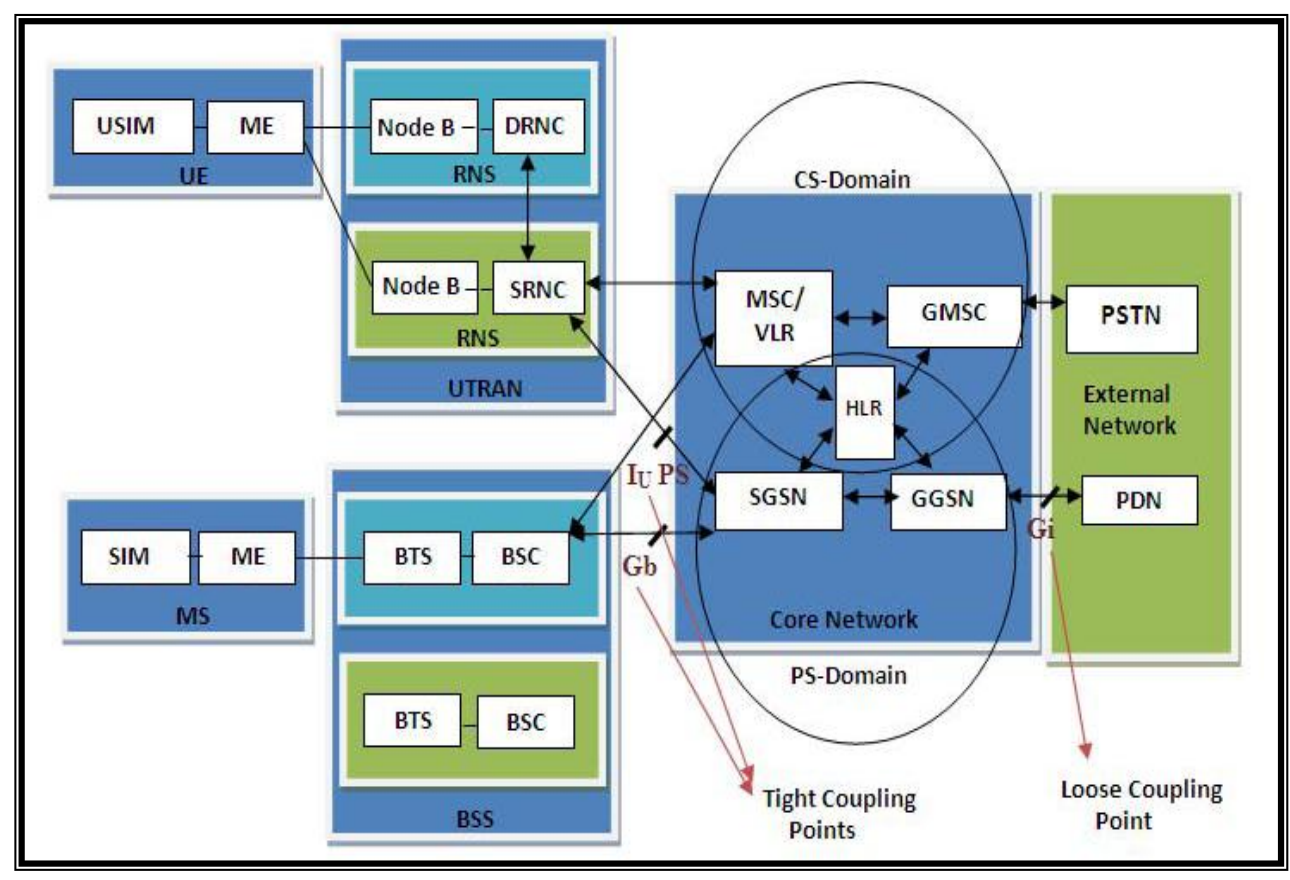

Fig 1: Tight and loose coupling points at UMTS network [9] 


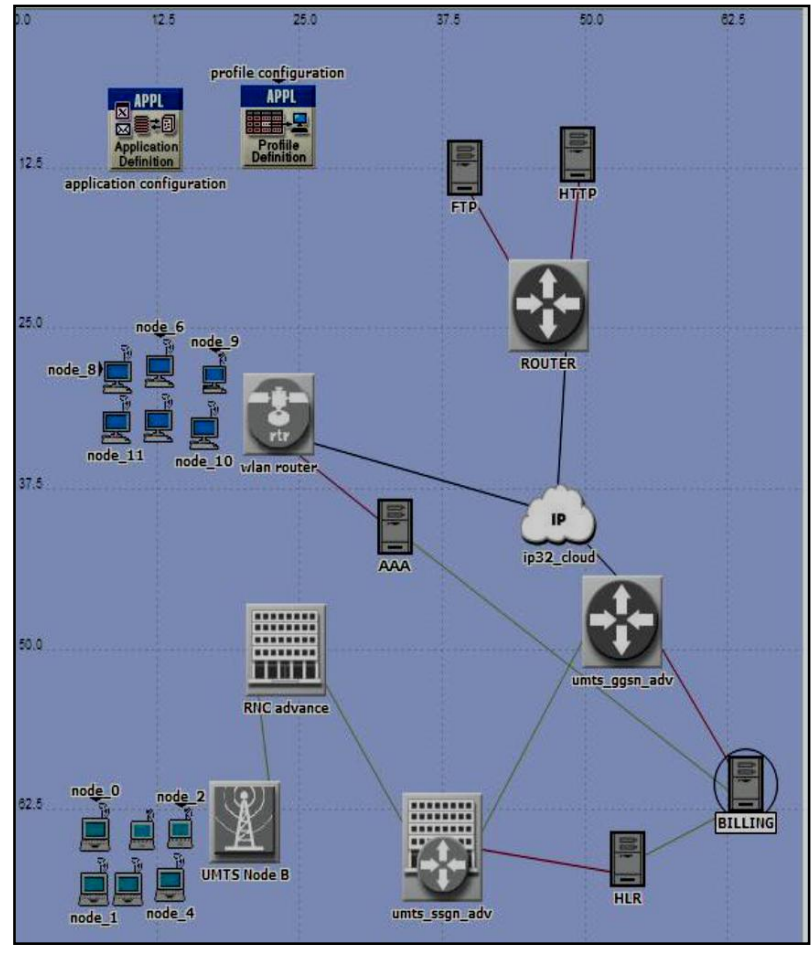

Fig 3: Open Coupling Scheme Architecture

We can clearly understand the difference in the networks of open and loose coupling scheme by having a look at the Figures 2 and 3 that is in case of open coupling scheme authentication server is separate for the two networks while in loose coupling scheme authentication is done by same server and billing is same for both of the schemes.

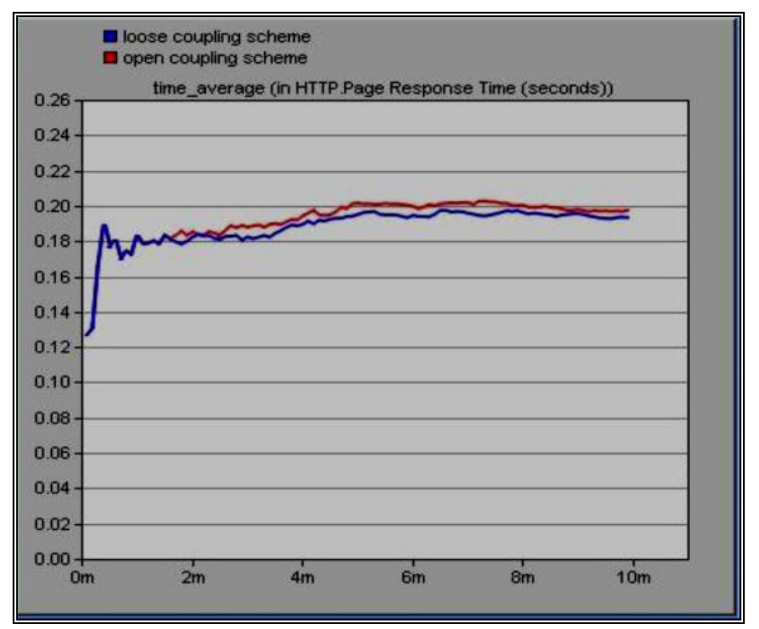

Fig 3: HTTP Page Response Time

Figure 3 shows the page response time for HTTP traffic. Horizontal axis shows the time for simulation as time increases from left to right number of user increases and vertical axis show the time for page response. It is clear from the figure when we have very little number of users at the start of the simulation time page response time is smaller and as we move left to right number of user increases and also it cause to increase the page response time. Blue color graph shows page response time for loose coupling architecture and red color graph shows the result for open coupling architecture, it is clear from the results that loose coupling architecture is better then open coupling. Figure 4 shows the traffic sent for HTTP request and it is clear in the figure that when number of users increases each user sends the request to have an access on the network to get HTTP so consequently data sent traffic increases.

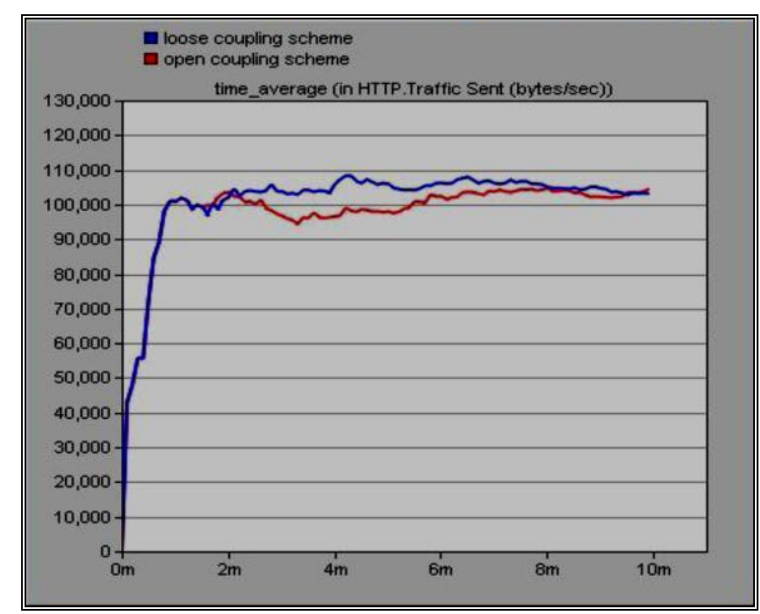

Fig 4: Http Traffic Sent

Figure 5 given below shows the graphs for FTP response time we consider the values in the graph after the spike where users actually send the request to download the file. It is clear from the graph that initially when there are few people who have started FTP download response time is low but as the simulation time passes more and more users send the FTP request which limits the networks resources and consequently it takes more response time. But if we compare response time for open and loose coupling architecture then loose coupling architecture is better because it has less response time. Figure 6 shows the traffic sent for FTP and as the user load increases with time data sent increases, open coupling scheme have to sent more number of bytes as compare to loose coupling scheme to get the internet applications. As we discuss in detail in chapter number five that in loose coupling architecture AAA server and packet switched services of UMTS are a part of IP cloud so that is why response time for internet applications is less and data sent is greater as compare to open coupling architecture in which only AAA is common.

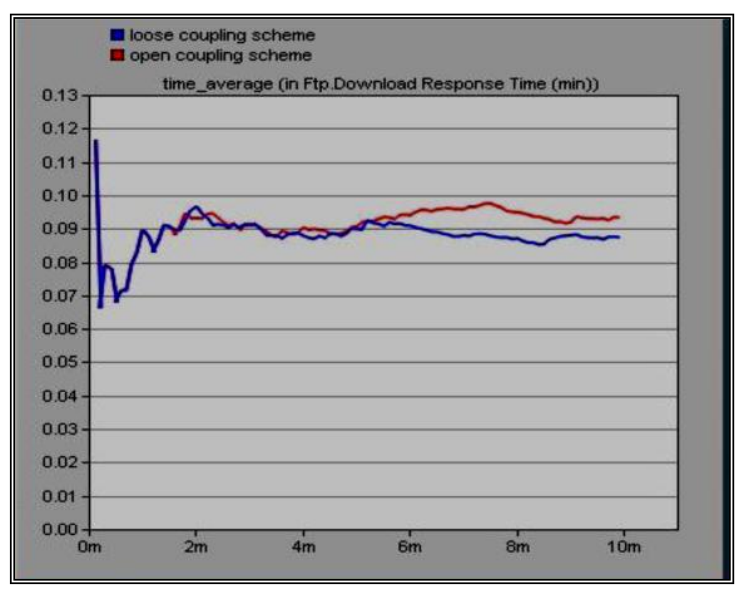

Fig 5: FTP Response Time 


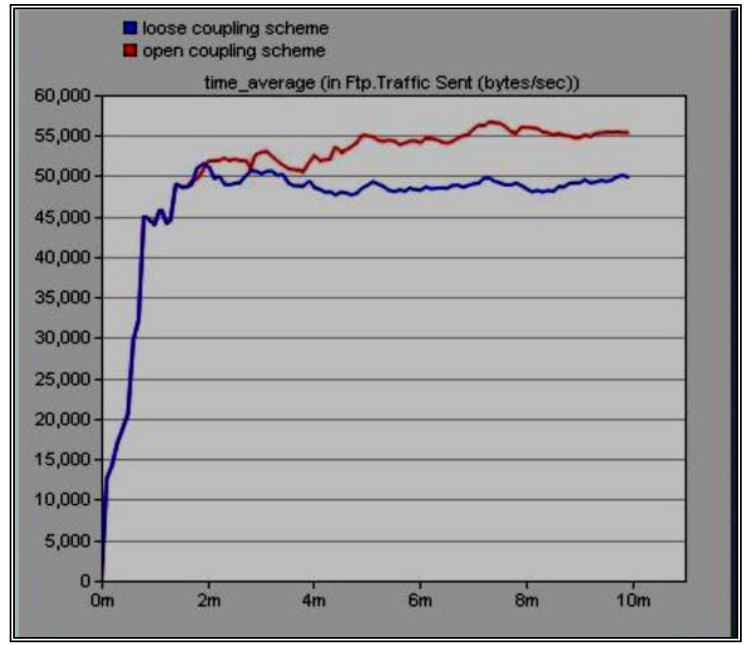

Fig 6: Ftp Traffic Sent

Graphs in Figure 7 shows that the delays which are offered by the whole network to the WLAN devices to initialize the internet services. The delay increases with the number of users and loose coupling architecture has smaller delay as compare to the open couple architecture. Graph in Figure 8 shows the data sent by the WLAN network which increases due to increase in number of users and open coupling architecture have to send more amounts of data in order to access same services as in loose coupling architecture. Graphs in the Figures 9 and 10 given below shows the graphs of traffic sent and traffic received In UMTS network while the users start moving from UMTS network to WLAN network, as users move to WLAN network very few people left in UMTS network and results in lower data sent and received and if we compare loose coupling to the open coupling data sent and received for UMTS network is higher in loose coupling architecture.

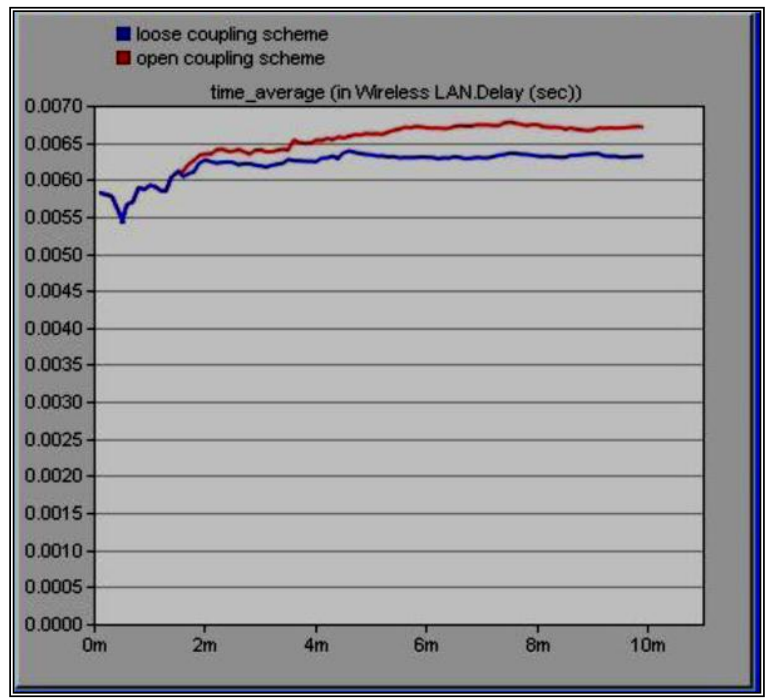

Fig 7: WLAN Delay

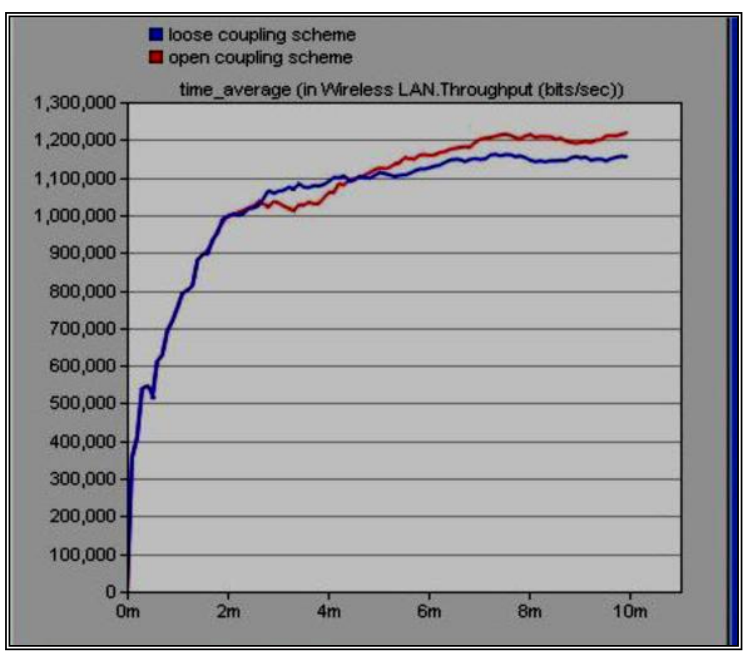

Fig 8: WLAN Throughput

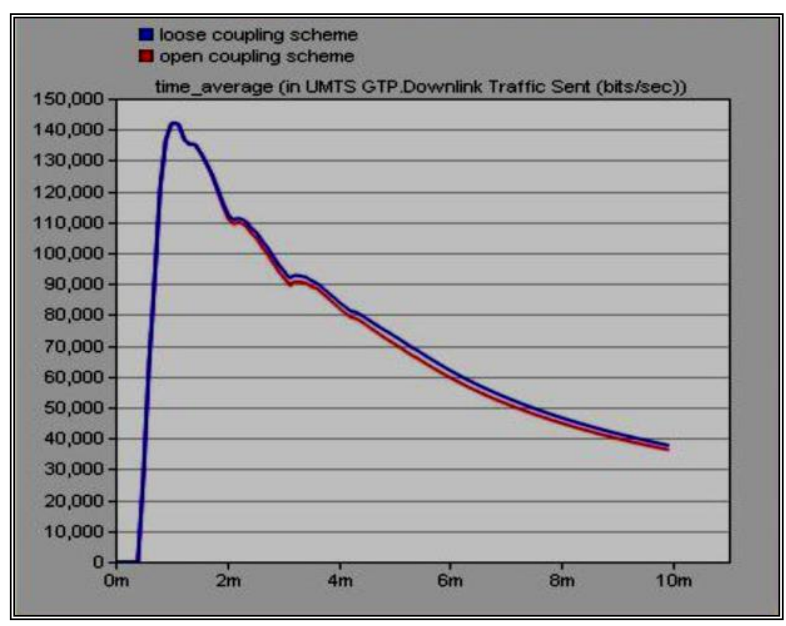

Fig 9: UMTS Downlink Traffic Sent

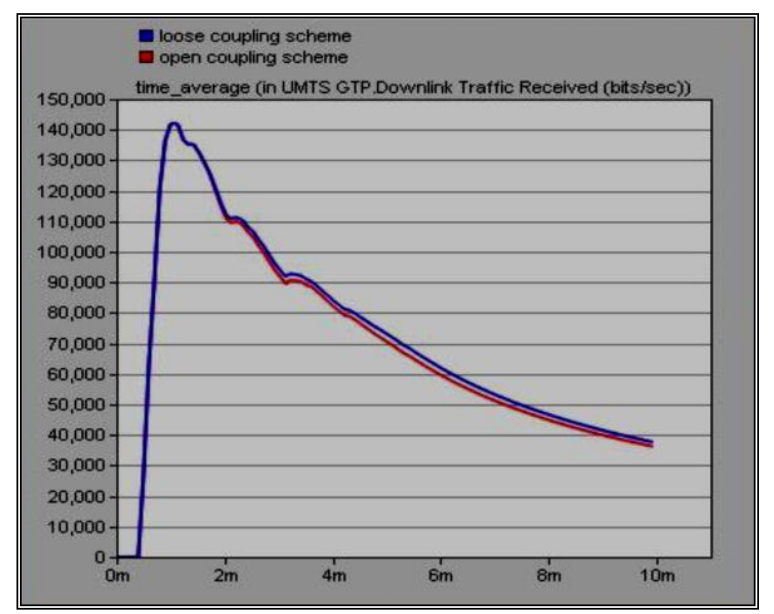

Fig 10: UMTS Downlink Traffic Received 


\section{CONCLUSIONS}

In this paper, several internetworking approaches for the integration of UMTS and WLAN networks were reviewed. The inherent differences between WLAN and UMTS enable enough technical challenges that need to be resolved in term of the integration between different networks for Next Generation Wireless Networks. These differences are in terms of protocols, algorithms, data rate, authentication mechanism, handover mechanism, coverage ranges, etc. To perform an integrated heterogeneous wireless network, several techniques have been proposed in the recent bibliography. Moreover, It was inclusively examined that two different techniques the open coupling schemes, the integration of the UMTS and WLAN, namely when both Billing and Authentication servers are common for UMTS and WLAN and the loose coupling Architecture, where only the billing Server is common for both networks WLAN and UMTS and each network use its own separate Authentication servers. The performance analysis of comparison of the loose coupling and open coupling architectures shows that loose coupling architecture is better than the open coupling architecture scheme in the case of HTTP and FTP, for all applied measurement parameters. The same result was found for the performance of the ubiquitous connectivity, namely by checking the WLAN access delay and UMTS delay, when the user is moving across the heterogeneous wireless networks.

\section{REFERENCES}

[1] H. Kaaranen et. al., "UMTS networks: Architecture, Mobility and Services", Wiley 2001.

[2] ETSI, "Requirements and Architectures for Interworking between HIPERLAN/2 and 3rd Generation Cellular Systems", Tech. rep. ETSI TR 101 957, Aug. 2001.

[3] 3GPP TR 22.934 V6.2.0, "Feasibility study on 3GPP system to Wireless Local Area Network (WLAN) interworking (Release 6)", September 2003.

[4] S.-L. Tsao, C.-C. Lin, "Design and evaluation of UMTS/WLAN interworking strategies", Vehicular Technology Conference, VTC 2002-Fall, 2002.

[5] V. Varma, S. Ramesh, K. D. Wong, M. Barton, G. Hayward and J. Friedhoffer, "Mobility Management in Integrated UMTS/WLAN Networks", Proceedings of ICC 2003, May 2003.

[6] D. Wong, M. Barton, B. Kim, V. Varma, S. Ramesh, G. Hayward, J. Friedhoffer, "UMTS Signaling over 802.11 Wireless LAN", VTC Fall 2003.

[7] R. Samarasinghe, V. Friderikos, A.H. Aghvami, "Analysis of Intersystem Handover: UMTS FDD \& WLAN", London Communications Symposium, 8th-9th September 2003.
[8] IST-2002-001858

http://www.everest-ist.upc.es

EVEREST project,

[9] Safdar Rizvi, N. M. Saad "A Dual Mode Mobile Terminal for Enhanced Performance of" Data Services in NGN Networks European Journal of Scientific Research ISSN 1450-216X Vol.67 No.3 (2012), pp. 360-377@) Euro Journals Publishing, Inc.2012,

[10] S. Glass, T. Hiller, S. Jacobs, and C. Perkins, "Mobile IP Authentication, Authorization, and Accounting Requirements," Internet draft (work in progress), 2000.

[11] IEEE 802.11 WG, "Wireless Medium Access Control (MAC) and physical layer (PHY) specifications: Medium Access Control (MAC) Enhancements for Quality of Service (QoS)," Technical Report, IEEE 802.11e/D3.0, 2002.

[12] S.Tsao and C.C. Lin. "Design and evaluation of UMTSWLAN interworking strategies". Proceedings of Vehicular Technology Conference. 2002

[13] A.K. Salkintzis. "WLAN/3G interworking architectures for next generation hybrid data networks". IEEE International Conference on Communications. 2004

[14] F. Siddiqui, S. Zeadally, and S. Fowler. "A Novel Architecture for Roaming between $3 \mathrm{G}$ and Wireless LANs".1st International Conference on Multimedia Services Access Networks, MSAN '05. 2005

[15] Y. Zhou, Y. Rong, H.A. Choi, J.H. Kim, J. K. Sohn, and H. In-Choi. "A Dual-mode Mobile Station Module for WLAN/UMTS Interworking Systems”, CiteSeerX, 2009

[16] M. Usman, W. Noshairwan, M. Gilani, A. Irshad and E. Irshad."Seamless vertical handoff using Authentication Certificate in GPRS-WLAN tightly coupled integrated networks". $4^{\text {th }}$ International Conference on emerging Technologies, ICET. 2008

[17] S. Minghui, H. Rutagemwa, X.Shen, J.W. Mark, A. Saleh. "A Service-Agent-Based Roaming Architecture for WLAN/Cellular Integrated Networks". IEEE Transactions on Vehicular Technology, 56(5): 3168 3181,2007

[18] R. B. Ali, S. Pierre. "On the impact of soft vertical handoff on optimal voice admission control in PCFbased WLANs loosely coupled to 3G networks". IEEE Transactions on Wireless Communications, 8(3):1356$1365,2009$.

[19] F. Zarai, N. Boudriga, M.S. Obaidat. "WLAN-UMTS Integration: Architecture, Seamless Handoff, and Simulation Analysis". SIMULATION, 82(6): 413-424, 2006. 